\title{
Statistics of photodissociation spectra: nonuniversal properties
}

\author{
Oded Agam \\ The Racah Institute of Physics, The Hebrew University, Jerusalem 91904, Israel.
}

\begin{abstract}
We consider the two-point correlation function of the photodissociation cross section in molecules where the fragmentation process is indirect, passing through resonances above the dissociation threshold. In the limit of overlapping resonances, a formula is derived, relating this correlation function to the behavior of the corresponding classical system. It is shown that nonuniversal features of the two-point correlation function may have significant experimental manifestations.
\end{abstract}

The photodissociation of molecules, such as the radicals $\mathrm{HO}_{2}$ and $\mathrm{NO}_{2}$, is an indirect process comprised of two steps. In the first step, a photon excites the molecule to an energy above the dissociation threshold. Then fragmentation proceeds via energy redistribution within the vibrational degrees of freedom, or tunneling from binding to unbinding energy surfaces of the adiabatic electronic potentialt. A barrier, which separates quasi-stable states from continuum modes, hinders the immediate dissociation of the excited molecule. On these long lived resonances, the dynamics of the system is chaotic. Therefore, the photodissociation cross section, exhibits a complicated behavior as function of the photon energye, behavior which suggests a statistical analysis of the problem. This appreach has been taken, recently, by Fyodorov and Alhassid 3 t who analyzed correlations of the photodisso-

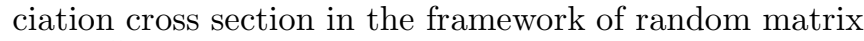
theory 5 . In this framework, one is able to describe the universal properties of the photodissociation cross section, but not the individual imprints of each molecule. It is well known, however, that the statistical properties of quantum chaotic systems bear a simple relation to the underlying classical dynamics 6 . The main purpose of this paper is to relate the statistical characteristics of the photodissociation cross section to correlation functions associated with the classical dynamics of the molecule.

Consider a molecule, in the ground state $|g\rangle$, excited by a light pulse to an energy above the dissociation threshold, and let $\mathcal{H}$ denote the Hamiltonian of the system on the excited electronic surface. It will be assumed that $\mathcal{H}$ represents an open system with several open dissociation channels. The photodissociation cross section of the molecule, in the dipole approximation, is given by

$$
\sigma(\epsilon)=-\eta \operatorname{Im}\left\langle g\left|\mathcal{D} \frac{1}{\epsilon^{+}-\mathcal{H}} \mathcal{D}\right| g\right\rangle
$$

where $\epsilon$ is the energy measured from the ground state of the molecule and $\epsilon^{ \pm}=\epsilon \pm i 0, \mathcal{D}=\mathbf{d} \cdot \hat{\mathbf{e}}$ is the projection of the electronic dipole moment operator of the molecule, $\mathbf{d}$, on the polarization, $\hat{\mathbf{e}}$, of the absorbed light, and $\eta=\epsilon / c \hbar \epsilon_{0}, c$ being the speed of light, and $\epsilon_{0}$ the electric permitivity.

We focus our attention on the dimensionless two-point correlation function:

$$
K(\omega)=\frac{\left\langle\sigma\left(\epsilon-\frac{\hbar \omega}{2}\right) \sigma\left(\epsilon+\frac{\hbar \omega}{2}\right)\right\rangle}{\langle\sigma(\epsilon)\rangle^{2}}-1,
$$

where $\langle\cdots\rangle$ denotes an energy averaging over a classically small energy interval centered at $\epsilon$ which, nonetheless, includes a large number of resonances. It will be assumed that the excitation energy, $\epsilon$, is sufficiently high such that the mean spacing between the vibrational modes of the molecule is smaller than the energy broadening due to the finite life time of the system in the excited states. This is the regime of overlapping resonances.

Turning to the classical counterpart of our system, let $\rho(\mathbf{x})$ be the initial density distribution of the excited molecule in phase space. Here $\mathbf{x}=(\mathbf{r}, \mathbf{p})$ is a phase space point, $\mathbf{r}$ and $\mathbf{p}$ being $d$ dimensional vectors of coordinates and conjugate momenta, respectively. The classical autocorrelation function of $\rho(\mathbf{x})$ is defined as

$$
C(t)=\frac{\langle\langle\rho(\mathbf{x}(t)) \rho(\mathbf{x})\rangle\rangle}{\langle\langle\rho(\mathbf{x})\rangle\rangle^{2}},
$$

where $\mathbf{x}(t)$ is the end point of the trajectory starting at $\mathbf{x}(0)=\mathbf{x}$, and evolving for a time $t$ according to the classical equations of motion: $\dot{\mathbf{r}}=\partial \mathcal{H} / \partial \mathbf{p}$ and $\dot{\mathbf{p}}=-\partial \mathcal{H} / \partial \mathbf{r}$. The averaging, $\langle\langle\cdots\rangle\rangle$, is over all initial points, $\mathbf{x}$, on the energy shell $\epsilon=\mathcal{H}(\mathbf{x})$ (see definition in Eq. (10) below). Another classical correlation function, closely related to $C(t)$, is defined by restricting the average in (3) only to those points $\mathbf{x}$ which lie on the periodic orbits of the system with period $\tau$, where $\tau \geq t$. This function will be denoted by $C_{\tau}(t)$, and its precise definition will be given later on (see Eq. (17)).

The central result of this paper is a formula which expresses the two-point correlation function of the photodissociation cross section, $K(\omega)$, in terms of the classical correlation functions, $C(t)$, and $C_{\tau}(t)$. Namely, within the semiclassical approximation, $K(\omega)$ takes the form

$$
K(\omega) \simeq K_{1}(\omega)+K_{2}(\omega)
$$

where

$$
\begin{aligned}
& K_{1}(\omega)=\frac{2}{\pi \beta \hbar} \operatorname{Re} \int_{0}^{\infty} d t e^{i \omega t} C(t), \quad \text { and } \\
& K_{2}(\omega)=\frac{1}{\pi^{2} \beta \hbar^{2}} \operatorname{Re} \int_{0}^{\infty} d t e^{i \omega t} \int_{-\frac{t}{2}}^{\frac{t}{2}} d \tau C_{t}(\tau) .
\end{aligned}
$$


Here $\beta=1$ for Hamiltonians with time reversal symmetry, and $\beta=2$ for systems without this symmetry. It is assumed that $\mathcal{H}$ have no other discrete symmetry.

The above results will be derived by semiclassical methods $\mathrm{B}$. The overlap of resonances implies that the behavior of $\sigma(\epsilon)$ is dominated by short time dynamics, and therefore the semiclassical approximation is justified. A semiclassical treatment of this problem will also clarify the role of various classical trajectories, and help interpreting formulae $(5-6)$.

In the semiclassical limit, the Green function is approximated by a sum of two terms: $G \simeq G_{W}+G_{\text {osc }}$. The first, known as the Weyl term, is a smooth function of the energy, $\epsilon$ :

$$
\left\langle\mathbf{r}^{\prime}\left|G_{W}\left(\epsilon^{ \pm}\right)\right| \mathbf{r}\right\rangle=\int \frac{d \mathbf{p}}{(2 \pi \hbar)^{d}} \frac{e^{\frac{i}{\hbar} \mathbf{p} \cdot\left(\mathbf{r}^{\prime}-\mathbf{r}\right)}}{\epsilon^{ \pm}-\mathcal{H}\left(\mathbf{p}, \frac{\mathbf{r}^{\prime}+\mathbf{r}}{2}\right)} .
$$

It is large when $\left|\mathbf{r}-\mathbf{r}^{\prime}\right|$ is of order of the particle wavelength, and therefore represents a local function in space. The second contribution, $G_{o s c}$, is nonlocal in space but oscillatory in the energy. It is expressed as a sum over classical trajectoriest:

$$
\left\langle\mathbf{r}^{\prime}\left|G_{o s c}\left(\epsilon^{ \pm}\right)\right| \mathbf{r}\right\rangle=\sum_{\nu} A_{\nu}^{ \pm} e^{ \pm \frac{i}{\hbar} S_{\nu}\left(\epsilon ; \mathbf{r}^{\prime}, \mathbf{r}\right)},
$$

where $S_{\nu}\left(\epsilon ; \mathbf{r}^{\prime}, \mathbf{r}\right)$ is the classical action of the $\nu$-th trajectory from $\mathbf{r}$ to $\mathbf{r}^{\prime}$ with energy $\epsilon$, and $A_{\nu}^{+}=\left(A_{\nu}^{-}\right)^{*}$ is the corresponding amplitude.

This decomposition of the Green function implies that the average, $\langle\sigma(\epsilon)\rangle$, comes only from the Weyl contribution, $G_{W}$. A straightforward calculation yields $\langle\sigma(\epsilon)\rangle=$ $\langle\langle\rho(\mathbf{x})\rangle\rangle$, where

$$
\rho(\mathbf{x})=\int d \mathbf{r}^{\prime} e^{\frac{i}{\hbar} \mathbf{p} \cdot \mathbf{r}^{\prime}}\left\langle\mathbf{r}-\frac{\mathbf{r}^{\prime}}{2} \mid \psi\right\rangle\left\langle\psi \mid \mathbf{r}+\frac{\mathbf{r}^{\prime}}{2}\right\rangle
$$

is the Wigner function of the (unnormalized) state $|\psi\rangle=$ $\mathcal{D}|g\rangle$. This function is real, and upon small smearing in phase space yields a positive definite function which can be interpreted as the initial density distribution of the excited molecule. The microcanonical average, $\langle\langle\rangle\rangle$, of a general function $f(\mathbf{x})$ is defined as

$$
\langle\langle f(\mathbf{x})\rangle\rangle=\int \frac{d \mathbf{x}}{(2 \pi \hbar)^{d}} \delta(\epsilon-\mathcal{H}(\mathbf{x})) f(\mathbf{x}) .
$$

Notice that $f$ and $\langle\langle f\rangle\rangle$ do not have the same dimensions. To simplify our notations, from now on we work in units where $\hbar=1$.

The correlator of $\sigma(\epsilon)$ can be written in the form:

$$
\begin{array}{r}
\left\langle\sigma\left(\epsilon-\frac{\omega}{2}\right) \sigma\left(\epsilon+\frac{\omega}{2}\right)\right\rangle=\frac{\eta^{2}}{2 \pi^{2}} \int \prod_{i=1}^{4} d \mathbf{r}_{i} \rho\left(\mathbf{r}_{1}, \mathbf{r}_{2}\right) \rho\left(\mathbf{r}_{3}, \mathbf{r}_{4}\right) \\
\times \operatorname{Re}\left\langle G\left(\epsilon^{-}-\frac{\omega}{2} ; \mathbf{r}_{2}, \mathbf{r}_{1}\right) G\left(\epsilon^{+}+\frac{\omega}{2} ; \mathbf{r}_{4}, \mathbf{r}_{3}\right)\right\rangle,
\end{array}
$$

where $\rho\left(\mathbf{r}, \mathbf{r}^{\prime}\right)=\langle\mathbf{r} \mid \psi\rangle\left\langle\psi \mid \mathbf{r}^{\prime}\right\rangle$. Considering the connected part of the correlator, only the oscillatory terms of the Green functions, $G_{o s c}$, contribute. Since these are strongly oscillating functions of the coordinates, the main contribution to the integral (11) comes from two types of orbits: (a) general orbits with initial and final points far apart, but $\mathbf{r}_{2} \sim \mathbf{r}_{3}$ and $\mathbf{r}_{1} \sim \mathbf{r}_{4}$. (b) Returning orbits with $\mathbf{r}_{1} \sim \mathbf{r}_{2}$ and $\mathbf{r}_{\mathbf{3}} \sim \mathbf{r}_{\mathbf{4}}$. The corresponding contributions to (11) will be denoted by $I_{a}$ and $I_{b}$, respectively. When $\mathcal{H}$ is also time reversal symmetric, additional contribution, equal to $I_{a}$, comes from orbits with $\mathbf{r}_{\mathbf{1}} \sim \mathbf{r}_{\mathbf{3}}$ and $\mathbf{r}_{2} \sim \mathbf{r}_{4}$.

The calculation of $I_{a}$ involves two main approximations. One is to expand the actions of the Green functions (8) to the first order in $\omega, \mathbf{r}_{3}-\mathbf{r}_{2}$, and $\mathbf{r}_{4}-\mathbf{r}_{1}$. For this purpose we use the relation: $S_{\nu}\left(\epsilon \pm \omega / 2 ; \mathbf{r}^{\prime}+\delta \mathbf{r}^{\prime}, \mathbf{r}+\delta \mathbf{r}\right) \simeq$ $S_{\nu}\left(\epsilon ; \mathbf{r}^{\prime}, \mathbf{r}\right) \pm t_{\nu} \omega / 2+\mathbf{p}_{\nu}^{\prime} \cdot \delta \mathbf{r}^{\prime}-\mathbf{p}_{\nu} \cdot \delta \mathbf{r}$, where $t_{\nu}$ is the time of the orbit, $\mathbf{p}_{\nu}$ is the initial momentum of the trajectory (at point $\mathbf{r}$ ), and $\mathbf{p}_{\nu}^{\prime}$ is the final momentum (at point $\mathbf{r}^{\prime}$ ). The second approximation is to neglect quantum interference effects (weak localization corrections) and employ the "diagonal approximation". In this approximation, the double sum on trajectories in the product of Green functions, $G_{o s c}\left(\epsilon^{+}\right) G_{o s c}\left(\epsilon^{-}\right) \simeq \sum_{\nu \nu^{\prime}} A_{\nu} A_{\nu^{\prime}}^{*} e^{i S_{\nu}-i S_{\nu^{\prime}}}$, is replaced by a single sum in which one keeps only pairs of trajectories, $\left(\nu, \nu^{\prime}\right)$, related by symmetry. Noticing that $\rho\left(\mathbf{r}_{1}, \mathbf{r}_{2}\right) \rho\left(\mathbf{r}_{3}, \mathbf{r}_{4}\right)=\rho\left(\mathbf{r}_{1}, \mathbf{r}_{4}\right) \rho\left(\mathbf{r}_{3}, \mathbf{r}_{2}\right)$, and integrating on the coordinate differences $\mathbf{r}_{3}-\mathbf{r}_{2}$, and $\mathbf{r}_{4}-\mathbf{r}_{1}$, one thus obtains:

$$
I_{a} \simeq \frac{\eta^{2}}{2 \pi^{2}} \operatorname{Re} \int d \mathbf{r} d \mathbf{r}^{\prime} \sum_{\nu}\left|A_{\nu}\right|^{2} \rho\left(\mathbf{x}_{\nu}^{\prime}\right) \rho\left(\mathbf{x}_{\nu}\right) e^{i t_{\nu} \omega},
$$

where $\mathbf{x}_{\nu}=\left(\mathbf{r}, \mathbf{p}_{\nu}\right)$ and $\mathbf{x}_{\nu}^{\prime}=\left(\mathbf{r}^{\prime}, \mathbf{p}_{\nu}^{\prime}\right)$ are the initial and final phase-space coordinates of the $\nu$-th trajectory.

Now, to evaluate the sum over $\nu$, we use the sum rule 10 :

$$
\begin{aligned}
& (2 \pi)^{d-1} \sum_{\nu}\left|A_{\nu}\right|^{2} g\left(\mathbf{x}_{\nu}^{\prime}, \mathbf{x}_{\nu}, t_{\nu}\right)= \\
& \int_{0}^{\infty} d t d \mathbf{p}^{\prime} d \mathbf{p} g\left(\mathbf{x}^{\prime}, \mathbf{x}, t\right) \delta(\epsilon-\mathcal{H}(\mathbf{x})) \delta\left(\mathbf{x}^{\prime}-\mathbf{x}(t)\right),
\end{aligned}
$$

where $g\left(\mathbf{x}^{\prime}, \mathbf{x}, t\right)$ is a general function of the time $t$, the initial $\mathbf{x}$, and the final $\mathbf{x}^{\prime}$ phase space points. $\mathbf{x}(t)$ is the phase space coordinate, after time $t$, of a trajectory starting from $\mathbf{x}(0)=\mathbf{x}$. Eq. (13) can be proved by performing the integrals on its right hand side. For this purpose it is convenient to use a local coordinate system with one coordinate parallel to the orbit, and $d-1$ perpendicular to it 10 . Substituting (13) in (12), and integrating over $\mathbf{x}$ yields

$$
I_{a} \simeq \frac{2 \eta^{2}}{(2 \pi)^{d+1}} \operatorname{Re} \int_{0}^{\infty} d t \int d \mathbf{x} e^{i t \omega} \delta(\epsilon-\mathcal{H}(\mathbf{x})) \rho(\mathbf{x}(t)) \rho(\mathbf{x}) .
$$

Using the definition of microcanonical average (10) and the correlation function (3), one obtains $K_{1}(\omega)=$ 
$2 I_{a} /\langle\langle\rho\rangle\rangle^{2} \beta$, where $\beta$ is the symmetry factor associated with the additional contribution in the case of time reversal symmetry.

To evaluate $I_{b}$, the term associated with returning trajectories, we expand the actions of the Green functions in (11), to first order in $\mathbf{r}_{2}-\mathbf{r}_{1}$ and $\mathbf{r}_{4}-\mathbf{r}_{3}$. Integrating over these coordinate differences, the result takes the form $I_{b}=\eta^{2} \operatorname{Re}\left\{I(\omega) I^{*}(\omega)\right\} / 2 \pi^{2}$, where

$$
I(\omega) \simeq \int d \mathbf{r} \sum_{\nu} A_{\nu} \rho\left(\mathbf{x}_{\nu}\right) e^{i S_{\nu}\left(\epsilon+\frac{\omega}{2} ; \mathbf{r}, \mathbf{r}\right)} .
$$

Here $\mathbf{x}_{\nu}=\left(\mathbf{r}, \overline{\mathbf{p}}_{\nu}\right)$ is a phase space point corresponding to the $\nu$-th returning trajectory at point $\mathbf{r}$, where $\overline{\mathbf{p}}_{\nu}=\left(\mathbf{p}_{\nu}^{\prime}+\mathbf{p}_{\nu}\right) / 2$ is the average of the initial, $\mathbf{p}_{\nu}$, and final, $\mathbf{p}_{\nu}^{\prime}$, momentum of the trajectory. The integral over $\mathbf{r}$ is now performed in the stationary phase approximation. The stationary phase condition, $\partial S_{\nu}(\epsilon ; \mathbf{r}, \mathbf{r}) / \partial \mathbf{r}=$ $\mathbf{p}_{\nu}-\mathbf{p}_{\nu}^{\prime}=0$, implies that the initial and final momentum of the orbit are equal, therefore, the main contribution to the integral (15) comes from the vicinities of periodic orbits11. The result can be expressed as a sum over periodic orbits: $I(\omega)=\sum_{p} A_{p} \rho_{p} e^{i S_{p}(\epsilon)+i t_{p} \omega / 2}$, where $A_{p}, t_{p}$ and $S_{p}$ are the amplitude, period, and action of the $p$-th periodic orbit respectively. $\rho_{p}=\oint d t \rho\left(\mathbf{x}_{p}(t)\right)$ is the time integral of $\rho(\mathbf{x})$ along the orbit $\mathbf{x}_{p}(t)$.

Next, we analyze the product $I(\omega) I^{*}(\omega)$, which in the diagonal approximation is given by $(2 / \beta) \sum\left|A_{p}\right|^{2} \rho_{p}^{2} e^{i t_{p} \omega}$. Following Eckhardt et al.12,

$$
\begin{gathered}
\rho_{p}^{2}=\int_{0}^{t_{p}} d \tau \int_{0}^{t_{p}} d \tau^{\prime} \rho\left(\mathbf{x}_{p}(\tau)\right) \rho\left(\mathbf{x}_{p}\left(\tau^{\prime}\right)\right)= \\
\int_{0}^{t_{p}} d t \int_{-\frac{t_{p}}{2}}^{\frac{t_{p}}{2}} d t^{\prime} \rho\left(\mathbf{x}_{p}\left(t-\frac{t^{\prime}}{2}\right)\right) \rho\left(\mathbf{x}_{p}\left(t+\frac{t^{\prime}}{2}\right)\right)= \\
=t_{p} \int_{-\frac{t_{p}}{2}}^{\frac{t_{p}}{2}} d t^{\prime}\left\langle\rho\left(\mathbf{x}\left(t^{\prime}\right)\right) \rho(\mathbf{x})\right\rangle_{p}
\end{gathered}
$$

where $\langle(\cdots)\rangle_{p}=t_{p}^{-1} \oint d t(\cdots)$ denotes the time average along the $p$-th periodic orbit. Thus $\left\langle I(\omega) I^{*}(\omega)\right\rangle=$ $(2 / \beta) \sum t_{p}\left|A_{p}\right|^{2} e^{i t_{p} \omega}\left\langle\rho\left(\mathbf{x}\left(t^{\prime}\right)\right) \rho(\mathbf{x})\right\rangle_{p}$. The sum over the periodic orbits can be converted into an integral over time using the relation

$$
\begin{aligned}
& \sum_{t<t_{p}<t+\delta t} t_{p}\left|A_{p}\right|^{2}\langle\cdots\rangle_{p}= \\
& \quad=\delta t \int d \mathbf{x} \delta[\epsilon-\mathcal{H}(\mathbf{x})] \delta\left[\mathbf{x}_{\|}-\mathbf{x}_{\|}(t)\right](\cdots),
\end{aligned}
$$

where $\mathbf{x}_{\|}$is a phase space coordinate on the energy shell $\mathcal{H}(\mathbf{x})=\epsilon$, while $\mathbf{x}_{\|}(t)$ is the position of the system after time $t$, starting from $\mathbf{x}_{\|}(0)=\mathbf{x}_{\|}$. Using (16), the sum over periodic orbits is converted into a time integral which yields formula (6) for $K_{2}(\omega)$, with the correlation function:

$$
C_{\tau}(t)=\int d \mathbf{x} \frac{\rho(\mathbf{x}(t)) \rho(\mathbf{x})}{\langle\langle\rho\rangle\rangle^{2}} \delta[\epsilon-\mathcal{H}(\mathbf{x})] \delta\left[\mathbf{x}_{\|}-\mathbf{x}_{\|}(\tau)\right] .
$$

The identification of the classical trajectories which underly the main contribution to the correlator $K(\omega)$, clarifies the origin of fluctuations in $\sigma(\epsilon)$. These have two sources: the wave functions of the system, and the density of states. $K_{1}(\omega)$, associated with general trajectories, is related to fluctuations in the wave functions, while $K_{2}(\omega)$, coming from the periodic orbits of the system, is related to fluctuations in the density of states.

To proceed further, one need to characterize the behavior of the correlation functions $C(t)$ and $C_{\tau}(t)$. For this purpose we assume that the classical dynamics of the system is characterized by two time scales which are well separated. Such a situation appears, for example, in almost close chaotic systems. There the long time scale is the typical time for dissociation, while the short time scale is the time which takes for a classical density distribution to relax to the ergodic distribution on the energy shell. We denote by $\mathcal{H}_{0}$ the closed Hamiltonian which controls the dynamics of the system for time shorter than the dissociation time. Under these assumptions $C(t) \simeq \Delta C_{t}(\tau) \simeq e^{-\gamma|t|} / \Delta$, where $\gamma$ is the dissociation rate, and $\Delta$ is the mean spacing between resonances, i.e. $(2 \pi \hbar)^{d} / \Delta=\int d \mathbf{x} \delta\left(\epsilon-\mathcal{H}_{0}(\mathbf{x})\right)$. We assume $\Delta$ to be approximately constant within the interval of energy averaging, and from now on work in units where $\Delta=1$. If $\Delta$ is not constant, $\sigma(\epsilon)$ should be unfolded appropriately.

An infinite separation between the time scales mentioned above corresponds to the limit of random matrix theoryt In this case $K(\omega)$ reduces to

$$
K_{u}(\gamma, \omega) \simeq \frac{2}{\beta \pi}\left(\frac{\gamma}{\omega^{2}+\gamma^{2}}+\frac{1}{2 \pi} \frac{\gamma^{2}-\omega^{2}}{\left(\gamma^{2}+\omega^{2}\right)^{2}}\right) .
$$

The first and the second terms of this formula come from $K_{1}(\omega)$ and $K_{2}(\omega)$ respectively. As can be seen, these components correspond to the leading and the subleading terms of an expansion in $1 / \gamma$.

Formula (18), describing the correlations in the universal regime, was first derived Fyodorov and AlhassidB using the nonlinear $\sigma$-model 13 . Here we confirm their conjecture that for overlapping resonances $(\gamma>1) K(\omega)$ can be derived by semiclassical methods.

Yet, the range of validity of formulae (4-6) goes far beyond the universal regime. They account also for system specific contributions to $K(\omega)$, which might be of the same order of the universal result (18). To be concrete, we focus on the nonuniversal corrections due to the leading term, $K_{1}(\omega)$, and consider a representative behavior of decay of correlations in open chaotic systems:

$$
C(t) \simeq e^{\gamma|t|}+\alpha e^{-\gamma_{2}|t|} \cos \left(\omega_{2} t\right) .
$$

Here $\gamma_{2}$ is the real part of the second Ruelle resonance 14 of the classical system, while $\omega_{2}$ is its imaginary part. $\alpha$ is a constant of order unity which depends on details of the system and the initial state (9). The situation where 
$\omega_{2} \neq 0$ appears usually when a specific short periodic orbit has strong influence on the dynamics of the system, since every typical trajectory stays in its vicinity for a long time. Thus, $\omega_{2} \approx 2 \pi / t^{*}$, where $t^{*}$ is the period of the orbit. Substitution of (19) in (4-6) yields:

$$
K(\omega) \simeq K_{u}(\gamma, \omega)+\frac{\alpha}{\pi \beta} \sum_{ \pm} \frac{\gamma_{2}}{\gamma_{2}^{2}+\left(\omega \pm \omega_{2}\right)^{2}}
$$

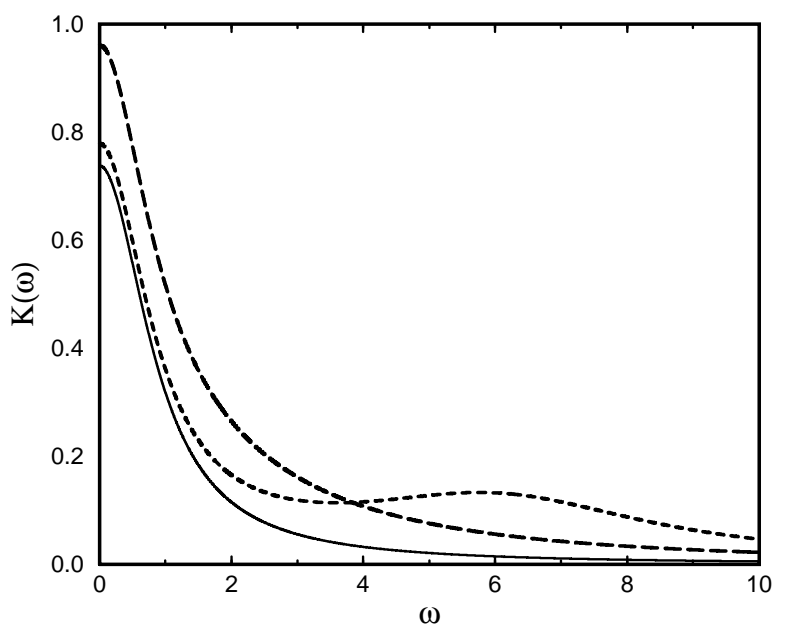

FIG. 1. The two-point correlation function of the photodissociation cross section for systems with time reversal symmetry $(\beta=1)$. The solid line is the universal result $K_{u}(\gamma, \omega)$ (Eq. 18) for the case $\gamma=1$. The dashed and the dotted lines correspond to the correlation function, $K(\omega)$, which includes the nonuniversal contribution, in the approximation of Eq. (20). In both cases $\gamma=1, \gamma_{2}=3$ and $\alpha=1$, but, the dashed line corresponds to $\omega_{2}=0$, while the dotted line to $\omega_{2}=6$.

This formula is plotted for various parameters in Fig. 1. The solid line represents the universal form (18). The dashed line, corresponding to $\omega_{2}=0$, shows the typical behavior of systems where the decay of correlations is of diffusive nature. In this case the main deviation from universality appears as an increase of correlations near the peak of $K(\omega)$, at $\omega=0$. The dotted line corresponds to a nonzero value of $\omega_{2}$, and characterizes the behavior of ballistic systems. Here the nonuniversal contribution is located in the tail of $K(\omega)$, near $\omega=\omega_{2}$, where the universal term (18) is already negligible. These plots demonstrate the significance of system specific contributions to $K(\omega)$.

In conclusion, we derived the semiclassical relation between the two point correlation function, $K(\omega)$, and the classical correlation functions $C(t)$, and $C_{\tau}(t)$. The first quantity characterizes the quantum mechanical process of photodissociation, while $C(t)$, and $C_{\tau}(t)$ characterizes the dynamics of the classical counterpart of the system. In contrast with the two-point correlation function of the density of states, where periodic orbits play the major role, here we have found that the main contribution to $K(\omega)$ comes from general orbits. Moreover, the nonuniversal contributions to the correlator, $K(\omega)$, can be of the same order of the universal result of random matrix theory (the relative magnitude being proportional to $\gamma / \gamma_{2}$ ). These results can be used in order to analyze the experimental data of complex molecules, extract information regarding their classical dynamics, and construct effective models for these molecules.

I would like to thank Yan Fyodorov for pointing out the problem of the semiclassical derivation of formula (18), and to Shmuel Fishman, Raphy Levine, and Nadav Shnerb for useful discussions and comments. This work was initiated at the "Extended Workshop on Disorder, Chaos and Interaction in Mesoscopic System" which took place in Trieste 1998. I thank the I.C.T.P. for the generous hospitality.

${ }^{1}$ R. Schinke, Photodissociation Dynamics, (Cambridge University, 1993)

${ }^{2}$ A. J. Dobbyn, M. Stumpf, H.-M. Keller, and W.L. Hase, J. Chem. Phys. 102, 8 (1995).

${ }^{3}$ Y. V. Fyodorov and Y. Alhassid, Phys. Rev. A 58, R3375 (1998).

${ }^{4}$ Y. Alhassid and Y. V. Fyodorov, J. Phys. Chem. 1029577 (1998).

${ }^{5}$ M. L. Mehta, Random Matrices, (Academic Press, New York, 1991)

${ }^{6}$ O Agam, B. L. Altshuler, and A. V. Andreev, Phys. Rev. Lett. 75, 4389 (1995).

${ }^{7}$ J. J. M. Verbaarschot, H. A. Weidenmüller, and M. R. Zirenbauer, Phys. Rep. 129, 367 (1985); Y. V. Fyodorov and H.-J. Sommers, J. Math. Phys. 38,1918 (1997).

${ }^{8}$ M. C. Gutzwiller, Chaos in Classical and Quantum Mechanics (Springer, New York, 1990).

${ }^{9}$ M. V. Berry and K. E. Mount, Rep. Prog. Phys. 35, 315 (1972).

${ }^{10}$ O. Agam, Phys. Rev. B. 54, 2607 (1996); and in Supersymmetry and Trace Formulae: Chaos and Disorder, (Plenum, 1999) pp. 133-151.

${ }^{11}$ M. C. Gutzwiller, J. Math. Phys. 8, 1979 (1967); 10, 1004 (1969); 11, 1791 (1970); 12, 343 (1971).

12 B. Eckhardt, S. Fishman, J. P. Keating, O. Agam, J. Main, and K. Müller, Phys. Rev. E. 52, 5893 (1995).

${ }^{13}$ K. B. Efetov, Adv. Phys. 32, 53 (1983).

${ }^{14}$ D. Ruelle, Phys. Rev. Lett. 56, 405 (1986). 